\title{
IMPROVED CORRECTION METHOD FOR WATER-REFRACTED TERRESTRIAL LASER SCANNING DATA ACQUIRED IN THE MOUNTAIN CHANNEL
}

\author{
N. Miura ${ }^{a *}$, Y. Asano $^{\text {a }}$, Y. Moribe ${ }^{b}$ \\ ${ }^{\text {a }}$ Graduate School of Agricultural and Life Sciences, the University of Tokyo, 1-1-1 Yayoi, Bunkyo-ku, Tokyo 113-8657 Japan - \\ (miura, yasano)@uf.a.u-tokyo.ac.jp \\ ${ }^{\mathrm{b}}$ Sanki Engineering Corporation, 3-8-24 Koshiba, Tobata-ku, Kitakyushu, Fukuoka Prefecture 804-0092 Japan - \\ moribe@sankieng.co.jp
}

Commission VIII, WG VIII/4

KEY WORDS: TLS, water refraction, acquisition, correction, underwater, point cloud

\begin{abstract}
:
Detailed information of underwater topography is required for better understanding and prediction of water and sediment transport in a mountain channel. Recent research showed promising utility of green-wavelength Terrestrial Laser Scanning (TLS) for measuring submerged stream-bed structure in fluvial environment. However, difficulty in acquiring reliable underwater data has been remained in the part of mountain channel where water surface has some gradient. Since horizontal water surface was a major premise for the existing water refraction correction method, significant error was resulted in such area. Therefore, this paper presents a modified method to correct water-refracted TLS data acquired over mountain channel with complex water-surface slope. Applicability of the modified method was validated using the field data and compared with the existing correction method and non-corrected data. The results showed that the modified method has much smaller error with RMSE value of $3 \mathrm{~mm}$ than the existing method (RMSE $=10$ $\mathrm{mm})$ and non-corrected data $(\mathrm{RMSE}=23 \mathrm{~mm})$. Presented method successfully corrected water-refracted TLS data acquired over sloped channel. This would enable us to quantitatively measure whole units of complex mountain channels, and help us to understand water dynamics better in the area.
\end{abstract}

\section{INTRODUCTION}

Detailed information of underwater topography is required for better understanding and prediction of water and sediment transport in a mountain channel. However, there were safety issues in surveying a steep mountain channel and lack of quantitative measurement methods was another obstacle to obtain such data. Recent research showed promising utility of green-wavelength Terrestrial Laser Scanning (TLS) for measuring submerged stream-bed structure in fluvial environment (e.g. Brasington et al., 2012; Hodge et al., 2009; Smith et al., 2012; Smith and Vericat, 2013). When light travels from air to water, it refracts (Figure 1). Refraction at the airwater interface causes systematic error to acquired TLS data. Smith et al (2012) developed a correction method based on Snell's Law for underwater data collected by TLS. The relationship between the angles of incidence $\theta_{a}$ and refraction $\theta_{w}$ of light passing through the boundary between the two media is described as

$$
\frac{\sin \theta_{\mathrm{a}}}{\sin \theta_{\mathrm{w}}}=n
$$

where $\mathrm{n}=$ refractive index

$\theta_{a}=$ angles of incidence

$\theta_{w}=$ refraction of light

The refractive index from air to clear water is approximately 1.33 and its variation for different temperatures is very small (Butler et al., 2002). However, TLS is not built specifically for through-water scanning and collected data takes no account of this refraction. Therefore, the authors calculated the actual position of underwater surveyed points from recorded locations of the points, the refractive index and the depth from scanner origin to water surface, which was assumed to be horizontal, using geometry of measurement and trigonometric function. With water refraction corrected data, the authors found that the introduced mean error was less than $5 \mathrm{~mm}$ through $200 \mathrm{~mm}$ of water in laboratory validation.

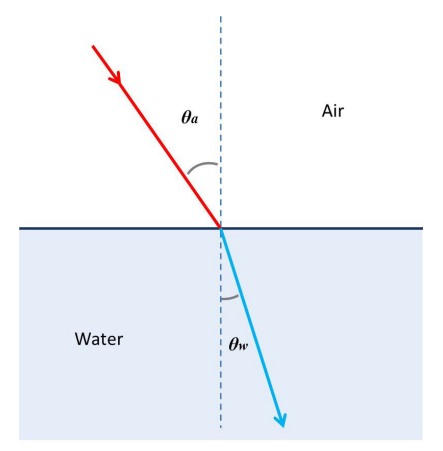

Figure 1. Refraction of light between air and water

For a steep mountain channel, TLS measurement has been a challenge since mountain channels are generally complex landforms and the channel morphology consists of variety of units such as cascades, steps, pools and other type of channel unit where most of bed materials are relatively small and

* Corresponding author 
submerged and the water surface has considerable gradient. Our previous research demonstrated good performance of greenwavelength TLS for acquiring underwater data in the pool unit of the channel down to a depth of approximately $70 \mathrm{~cm}$ (Miura and Asano, 2013). However, difficulty in acquiring reliable underwater data has been remained in the part of mountain channel where water surface has some gradient (Miura and Asano, 2015). In particular, the existing water-refraction correction method was not applicable in such area because horizontal water surface was a major premise for the method. Under such premise, water-refraction correction works too less in upper stream and too much in lower stream where watersurface slope exists. Therefore, this paper focuses on improving the existing water-refraction correction method which can be applied to the area where water-surface slope is complex. First, the detail of the modified method is described. Then, applicability of the modified method is validated using the field data and compared with the existing correction method and non-corrected data.

\section{MATERIAL AND METHODS}

\subsection{Study area}

The mountain channel we studied locates in the Hirudani Experimental basin $\left(0.82 \mathrm{~km}^{2}\right)$ at the Hodaka Sedimentation Observatory of the Disaster Prevention Research Institute, at Kyoto University in Gifu, central Japan $\left(36^{\circ} 25^{\prime} \mathrm{N}, 137^{\circ} 58^{\prime} \mathrm{E}\right)$. The mean annual air temperature is $9.5^{\circ} \mathrm{C}$. The mean annual precipitation is $1980 \mathrm{~mm}$, a quarter of which falls as snow in winter (Masaoka et al., 2016). Since the basin includes the active volcano, Mt. Yakedake, channels are characterized with large sediment transport. Study section of the channel is approximately $13.5 \mathrm{~m}$ long and located just downstream of the check dam for discharge and measurement of sedimentation rate (Figure 2). Approximately half the sediment trapped by the check dam was discharged with snow melt from the score gate 18 days before our survey in June 2015. The bed slope of the studied channel section was $9 \%$. The active channel width and wetted width ranged from $3.5 \mathrm{~m}$ to $4 \mathrm{~m}$ and from $1.05 \mathrm{~m}$ to 1.87 $\mathrm{m}$ respectively with water depth ranged from $0.06 \mathrm{~m}$ to $0.15 \mathrm{~m}$. $\mathrm{D}_{30}, \mathrm{D}_{50}$ and $\mathrm{D}_{84}$, which represent the 30th, 50th and 84th percentiles of the cumulative grain size distribution of riverbed materials, were $0.035 \mathrm{~m}, 0.06 \mathrm{~m}$ and $0.16 \mathrm{~m}$.

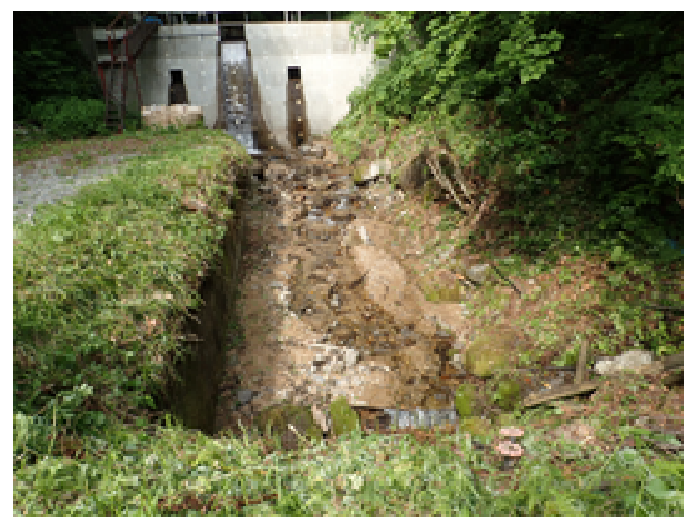

Figure 2. Study section of the mountain channel

\subsection{Field survey data}

Field survey was conducted in June 2015. Seven surveying poles were levelled and placed in the channel where water- surface slope changes. The water depth and the depth from channel bed to the top of poles were manually measured and recorded. The location of the poles was also recorded using Leica TS30 total station with prism and detail pole. Additionally, 28 points in the channel were surveyed for water depth and location.

\subsection{Terrestrial Laser Scanning}

TLS data was acquired using Leica ScanStation C10 over the study area. The acquisition was synchronized with the field survey. The sensor is operated with $532 \mathrm{~nm}$ pulsed laser. The accuracy of single measurement is $6 \mathrm{~mm}$ for position, $4 \mathrm{~mm}$ for distance and $60 \mu \mathrm{rad}$ for angle (Leica Geosystems, 2013). Multiple measurements were conducted by positioning TLS at 6 different locations surrounding the study section with minimum point spacing of $5 \mathrm{~mm}$ at $10 \mathrm{~m}$ (Figure 3). All data were collected relatively short ranges $(<20 \mathrm{~m})$. We followed the acquisition protocol outlined by Miura and Asano (2015). For data processing of the acquired TLS data, Leica software program, Cyclone 8.1, generic CAD and Bentley Systems, MicroStation V8 were used.

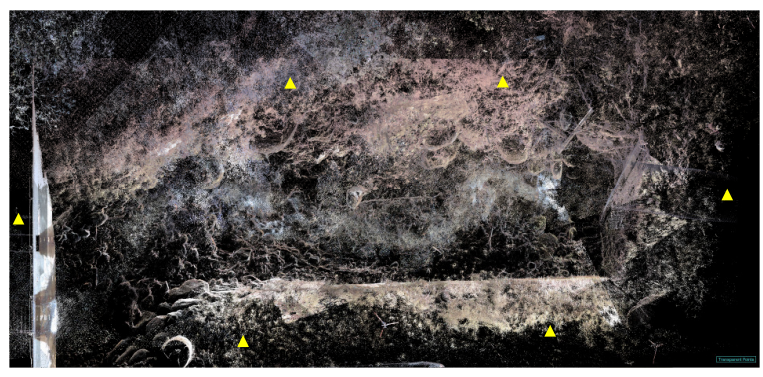

Figure 3. TLS data of study section. Check dam locates in the left side of the figure and triangles show scanner locations.

\subsection{Modified water refraction correction}

2.4.1 Segmentation: The study section was first segmented roughly into 8 blocks (Block1-8) which have similar watersurface slope using photographs of the area. Then, the boundaries between the blocks were adjusted referencing horizontally sliced section of acquired TLS data, which are combined point cloud converted from Cartesian coordinates to geographic coordinates, over the blocks (Figure 3). In this paper, detailed method for water refraction correction is described using Block 8 data.

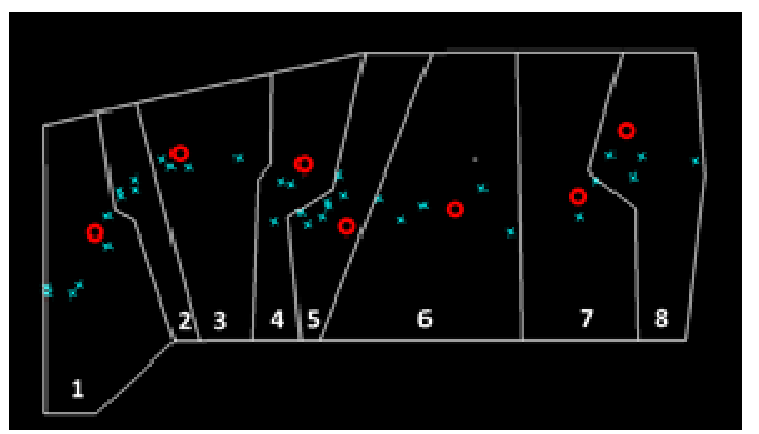

Figure 3. Segmented blocks (Block 1-8). Check dam locates in the left side of the figure. Circles show locations of surveying poles and $\mathrm{x}$-marks are surveyed points. 
2.4.2 Identification of water-surface slope: To identify watersurface slope in each block, survey points were used. Each block contains more than 3 survey points which has location, that is, coordinate and water depth information. Water-surface slope was identified by creating a triangle by connecting 3 survey points at water-surface level in each block (Figure 4a). Then, a rectangular surface model whose longer sides are parallel with water flow direction was created from the triangle in each block (Figure 4b). The longer side of bottom face is defined as $x^{\prime}$ axis and the direction perpendicular to the watersurface slope as z' axis.

2.4.3 Coordinate transformation of TLS data: In order to correct water refracted TLS data, the point cloud in each measurement were converted back to Cartesian coordinates which have scanner origins as Smith et al. (2012) suggested. Next, $\mathrm{x}$ and $\mathrm{z}$ axes of the point cloud were rotated on the scanner origin to fit with $x^{\prime}$ and $z$ ' axes of the rectangular surface model (Figure 5). This creates a new dataset with horizontal water-surface relative to the point cloud. The depth from the scanner origin to the water-surface was calculated. Then, water refraction correction (Smith et al., 2012) was applied to the underwater data. Finally, water-refraction corrected point cloud were rotated back to the original $\mathrm{x}$ and $\mathrm{z}$ axes and converted again to geographic coordinates. This process was repeated for the point cloud in each measurement.

2.4.4 Validation: In order to validate the applicability of the modified correction method, the accuracy of existing, modified and non-correction methods were compared. Triangular Irregular Network (TIN) for channel bed was created using the point cloud of each measurement in each method. The depth from channel bed to the top of pole was calculated using TIN and coordinate of the pole. These values were statistically compared to the manually measured depth.

\section{RESULTS}

Rotated angle of $\mathrm{x}$ and $\mathrm{z}$ axes of the point cloud in each measurement, which was utilized in the modified method, was summarized in Table 1. Rotated angle of $x$ axis varied among scanner locations; however, the angle of $\mathrm{z}$ axis was the same. Figure 6 displayed water-refraction corrected area in the point cloud of existing and modified methods. Upper stream area was less corrected in the existing method than in the modified method. Submerged point cloud seemed to be successfully identified in the modified method when it was inspected with a photograph in the same area. Figure 7 showed comparison of depth from channel bed to the top of pole among existing, modified and non-correction methods. Modified method was the closest to the manually measured depth followed by existing and non-correction method in this order. Root Mean Squair Error (RMSE) values against the manually measured depth were $3 \mathrm{~mm}$ for modified, $10 \mathrm{~mm}$ for existing and $23 \mathrm{~mm}$ for noncorrection methods. The difference from the manually measured depth in modified method was significantly smaller than that in existing method (paired $t$-test, $t=3.32, p<0.05$ ).

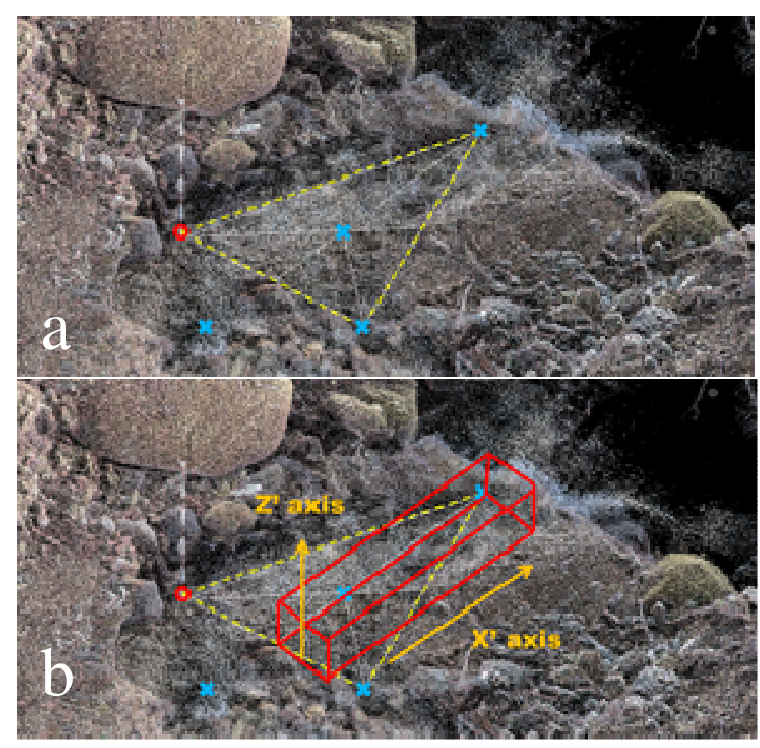

Figure 4. (a) Triangle created from surveyed points and a pole in Block 8, (b) a rectangular surface model.

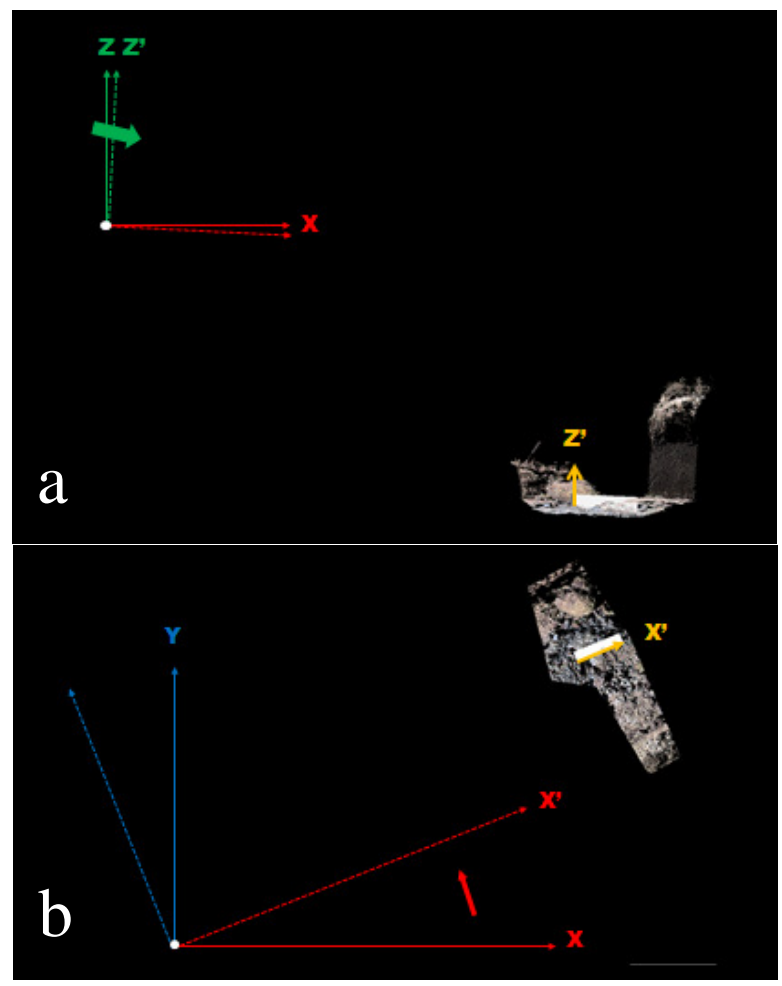

Figure 5. Rotation of $\mathrm{z}$ axis (a) and $\mathrm{x}$ axis (b) of the point cloud on the scanner origin.

\begin{tabular}{|c|c|c|}
\hline Scanner location & \multicolumn{2}{|c|}{ Rotation angle } \\
\hline & $\mathrm{X}$ axis & $\mathrm{Z}$ axis \\
\hline Measurement 1 & L $22.4^{\circ}$ & $\mathrm{R} 2.3^{\circ}$ \\
\hline Measurement 2 & L $175.4^{\circ}$ & R $2.3^{\circ}$ \\
\hline Measurement 3 & $\mathrm{~L} 41^{\circ}$ & R $2.3^{\circ}$ \\
\hline Measurement 4 & $\mathrm{~L} 96.3^{\circ}$ & $\mathrm{R} 2.3^{\circ}$ \\
\hline Measurement 5 & $\mathrm{R} 124.1^{\circ}$ & R $2.3^{\circ}$ \\
\hline Measurement 6 & $\mathrm{R} 56.5^{\circ}$ & $\mathrm{R} 2.3^{\circ}$ \\
\hline
\end{tabular}

Table 1. Rotated angle of $\mathrm{x}$ and $\mathrm{z}$ axes of TLS data. Direction of rotation is shown as $\mathrm{R}$ (clockwise) and $\mathrm{L}$ (anticlockwise). 


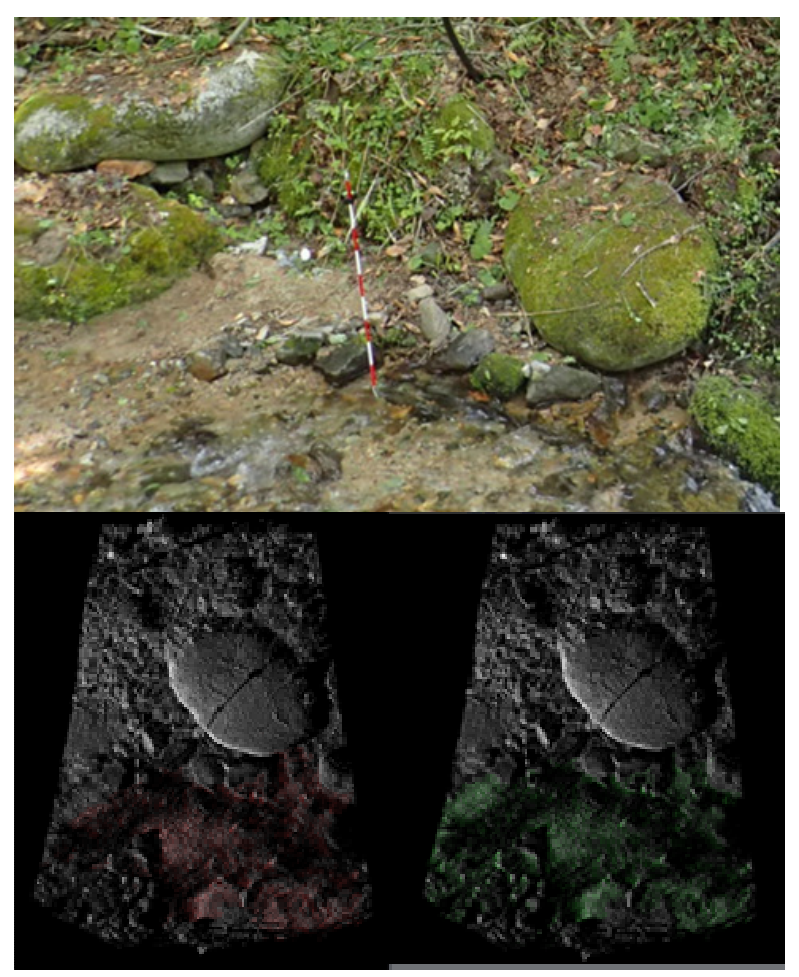

Figure 6. Comparison of water-refraction corrected area in the point cloud of the existing (bottom left) and modified (bottom right) methods, and a photograph in the same area (top). Water flows from left side to right side of the figure.

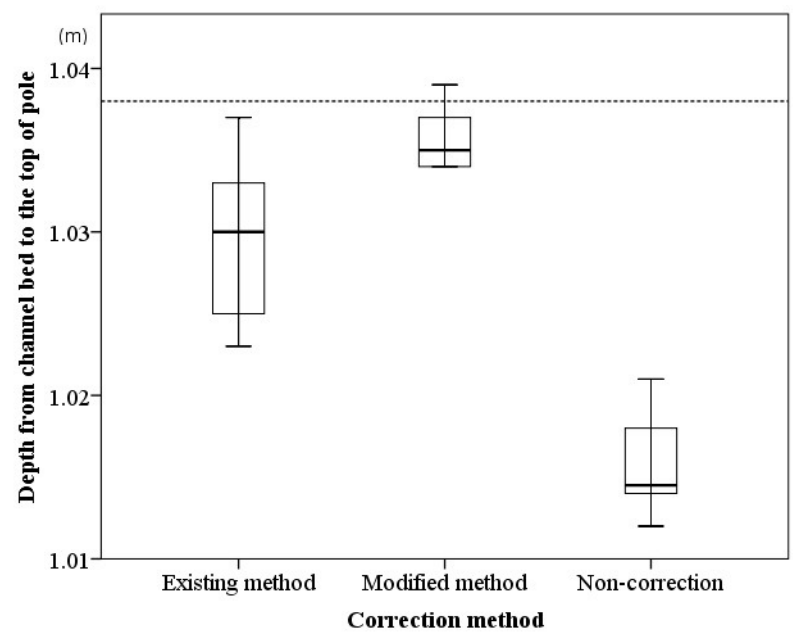

Figure 7. Comparison of depth from channel bed to the top of pole among correction methods. The broken line shows manually measured depth.

\section{DISCUSSION}

In the process of modified correction method, the point cloud was rotated on the scanner origin to eliminate the negative effect of water-surface slope. Rotation angle of $\mathrm{z}$ axis was constant in the block since the relationship between $\mathrm{z}$ axis of the scanner and the water-surface slope was the same regardless of the scanner locations. Comparison of water-refraction corrected area in the point cloud of the existing and modified methods clearly showed negative effect of water-surface slope on the existing correction method, which is undercorrection in upper stream and excess correction in lower stream of the channel (Figure 6). The modified method, which created the new dataset with horizontal water-surface relative to the point cloud by rotating the point cloud for water-refraction correction, worked well in eliminating the negative effect of the slope and identifying submerged point cloud. The results of statistics present that the modified method indeed has much smaller error than the existing method and non-corrected data in calculating the depth from channel bed to the top of pole. This is because TLS points in submerged channel bed area were properly waterrefraction corrected and moved to their true position. Presented method successfully corrected water-refracted TLS data acquired over sloped channel. This would enable us to quantitatively measure whole units of complex mountain channels, and help us to understand water dynamics better in the area.

\section{ACKNOWLEDGEMENTS}

This research was supported by JSPS KAKENHI Grant Number 26340001. We thank the staff of the Hodaka Sedimentation Observatory of the Disaster Prevention Research Institute, Kyoto University for their support.

\section{REFERENCES}

Brasington, J., Vericat, D., Rychkov, I., 2012. Modeling river bed morphology, roughness, and surface sedimentology using high resolution terrestrial laser scanning. Water Resources Research 48, W11519.

Butler, J., Lane, S., Chandler, J., Porfiri, E., 2002. ThroughWater Close Range Digital Photogrammetry in Flume and Field Environments. The Photogrammetric Record 17, 419-439.

Hodge, R., Brasington, J., Richards, K., 2009. In situ characterization of grain-scale fluvial morphology using Terrestrial Laser Scanning. Earth Surface Processes and Landforms 34, 954-968.

Leica Geosystems, 2013. Leica ScanStation C10.

Masaoka, N., Kosugi, K.i., Yamakawa, Y., Tsutsumi, D., 2016. Processes of bedrock groundwater seepage and their effects on soil water fluxes in a foot slope area. Journal of Hydrology 535, $160-172$.

Miura, N., Asano, Y., 2013. Green-wavelength terrestrial laser scanning of mountain channel. ISPRS Ann. Photogramm. Remote Sens. Spatial Inf. Sci. II-5/W2, 187-192.

Miura, N., Asano, Y., 2015. Effective Acquisition Protocol of Terrestrial Laser Scanning for Underwater Topography in a Steep Mountain Channel. River Research and Applications, doi: 10.1002/rra.2986.

Smith, M., Vericat, D., Gibbins, C., 2012. Through-water terrestrial laser scanning of gravel beds at the patch scale. Earth Surface Processes and Landforms 37, 411-421.

Smith, M.W., Vericat, D., 2013. EVALUATING SHALLOWWATER BATHYMETRY FROM THROUGH-WATER TERRESTRIAL LASER SCANNING UNDER A RANGE OF HYDRAULIC AND PHYSICAL WATER QUALITY CONDITIONS. River Research and Applications 30, 905-924. 